\title{
First International Workshop on Conceptual Modelling of Service (CMS 2010)
}

\section{Preface}

The aim of the First International Workshop on Conceptual Modelling of Service (CMS)" was to bring together researchers in the areas of services computing, services science, business process modelling, and conceptual modelling. The emphasis of this workshop is on the intersection of the rather new, fast growing services computing and services science paradigms with the well established conceptual modelling area.

The call for papers solicited submissions addressing modelling support for service integration; quality of service modelling; modelling languages / techniques for services; conceptual models for integrated design and delivery of value bundles; modelling of semantic services; and formal methods for services computing / services science.

A total number of 17 submissions were received by the workshop. Each paper was reviewed by three or four members of the international programme committee, and we finally selected the five best rated submissions for presentation at the workshop. The accepted papers were organised in two sessions. In the first session focused on Modelling Support for Service Integration while the second session covered Modelling Techniques for Services.

We wish to thank all authors of submitted papers and all workshop participants, without whom the workshop would not have been possible. We are grateful to the members of the programme committees for their timely expertise in carefully reviewing the submissions. Finally, our thanks go to the organizers of ER 2010 and the workshop chairs Gillian Dobbie and Juan Trujillo for giving us the opportunity to organise this workshop. 